Research Article

\title{
Finite Element Stress Model of Direct Band Gap Ge Implementation Method Compatible with Si Process
}

\author{
Xiaohuan Xue $\mathbb{D}^{\mathrm{D}}$, Jianjun Song $\mathbb{D}^{\mathrm{D}}$, and Rongxi Xuan \\ Key Lab of Wide Band-Gap Semiconductor Materials and Devices, School of Microelectronics, Xidian University, \\ Xi'an 710071, China \\ Correspondence should be addressed to Xiaohuan Xue; 1653115951@qq.com
}

Received 10 April 2019; Accepted 7 August 2019; Published 16 September 2019

Academic Editor: Sergio E. Ulloa

Copyright ( $\odot 2019$ Xiaohuan Xue et al. This is an open access article distributed under the Creative Commons Attribution License, which permits unrestricted use, distribution, and reproduction in any medium, provided the original work is properly cited.

As an indirect band gap semiconductor, germanium $(\mathrm{Ge})$ can be transformed into a direct band gap semiconductor through some specific modified methods, stress, and alloying effect. Direct band gap-modified Ge semiconductors with a high carrier mobility and radiation recombination efficiency can be applied to optoelectronic devices, which can improve the luminous efficiency dramatically, and they also have the potential application advantages in realizing monolithic optoelectronic integration (MOEI) and become a research hotspot in material fields. Among the various implementations of Ge band gap-type conversion, the related methods that are compatible with the Si process are most promising. It is such a method to etch around the Ge epitaxial layer on the Si substrate and introduce the biaxial tensile stress by SiGe selective filling. However, the influence of the width of the epitaxial layer, Ge composition, and Ge mesa region width on strain distribution and intensity is not clear yet. Accordingly, a finite element stress model of the selective epitaxy-induced direct band gap Ge scheme is established to obtain the material physical and geometric parameters of the $\mathrm{Si}_{1-x} \mathrm{Ge}_{x}$ growth region. The result of finite element simulation indicates when the $\mathrm{Si}_{1-x} \mathrm{Ge}_{x}$ epitaxial layer is $150-250 \mathrm{~nm}$ wide and the Ge composition is $0.3 \sim 0.5$, Ge mesa with $20-40 \mathrm{~nm}$ in width can be transformed into direct band gap semiconductors in the depth of $0-6 \mathrm{~nm}$. The theoretical results can provide an important theoretical basis for the realization of subsequent related processes.

\section{Introduction}

$\mathrm{Ge}$ is an indirect band gap semiconductor. Its direct band gap is only $0.14 \mathrm{eV}$ larger than the indirect band gap at room temperature. Ge can be transformed into a direct band gap material under certain modification conditions. "Modification" refers to the conduction band valley at the center of the Ge Brillouin zone, and the conduction band valley in the direction of [111] at the boundary of the Brillouin zone is gradually decreased with respect to the valence band top through some specific methods and techniques [1]. It is also required that the decreasing rate of the conduction band valley at the center of the Ge Brillouin zone is higher than that of the [111] direction. Therefore, Ge can be transformed into a quasidirect or a direct band gap material under certain conditions. Modified Ge has great potential for applications in optoelectronic and MOEI devices due to its energy level splitting, reduced effective mass, and high carrier mobility and radiation recombination efficiency.

Figure 1 shows various components required for MOEI, such as electronics, LED devices, and detectors. The realization of optoelectronic interconnection requires simultaneous integration of both electrical and optical devices on a single chip [2-4]. However, the current research on materials for high-efficiency light-emitting devices has been limited. The main reason is that III-V materials are costly and have a small application range in material preparation and device fabrication, and IV semiconductors, which are widely studied, are all indirect band gap materials [5], so it is necessary to find a semiconductor material suitable for use as a Si-based light source. In recent years, modified Ge materials have become a research hotspot for photoelectric integration. On the one hand, modified Ge has excellent hole mobility and can be used in high-speed semiconductor 


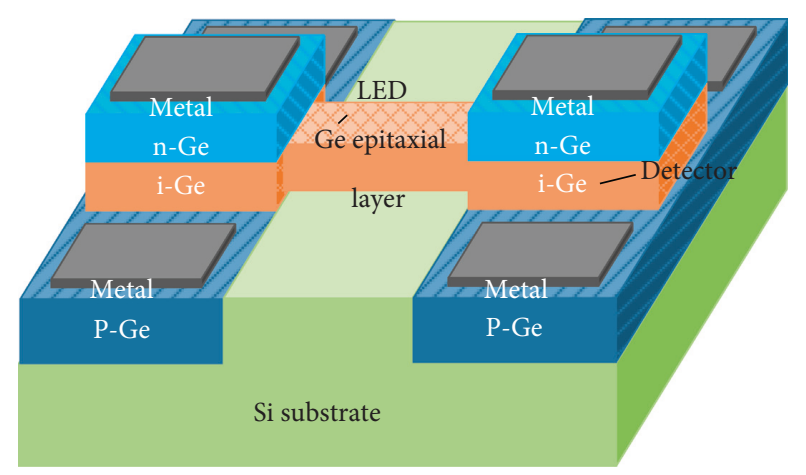

Figure 1: A diagram view of the MOEI device.

electrical devices [6]. On the other hand, modified Ge has a quasi-direct band gap structure, which can realize direct band gap luminescence by means of energy band engineering and other means and thus can be applied to lightemitting devices such as lasers and LEDs [7-16]. Most importantly, both Ge and Si belong to IV materials and have significant advantages in process and production costs.

Despite its wide application prospects, the preparation of modified Ge is facing many difficulties [17]. Since the quality is largely affected by the crystallization situation, structural defects, dislocations, and components, the preparation process of modified Ge has become one of the focuses in the basic research of this material. At present, the modified Ge preparation methods mentioned in the literature mainly include tensile strain-induced Ge direct band gap technology and GeSn alloying-induced direct band gap technology [18]. Based on the theory of strain tensor and deformation potential, the physical mechanism of Ge band gap conversion is investigated, which reveals the law that Ge transitions from indirect band gap semiconductors to direct one $[19,20]$. The results show the following: (1) The transformation can be achieved when the (001) biaxial tensile stress of the Ge material is about $2.4 \mathrm{Gpa}$. Figures 2 and 3 show the variation of the energy levels of Ge conduction and the strain generated with the stress applied under (001) biaxial tensile stress, respectively. Under (101) and (111) biaxial stresses, the transformation of the Ge band gap type cannot be realized. (2) For the uniaxial stress, the Ge semiconductor can be converted into a direct band gap semiconductor only under the (001) plane uniaxial $0^{\circ}$-[100] crystal-orientation tensile stress and the requiring stress is about $4.8 \mathrm{GPa}$ [21]. (3) In the Sn alloying scheme, Ge is changed to direct band gap when the Sn component is dissolved in Ge by about $8 \%$ $[22,23]$.

At present, the direct band gap technique of GeSn alloying has the problems of low solid solubility of Sn in Ge (about 1\%), serious surface segregation, and incompatibility with the Si process, and the tensile strain-induced direct band gap Ge technology has the defects of difficult process realization, many crystal defects, poor quality, and so on [24]. To this end, this paper uses a direct band gap Ge implementation method compatible with the $\mathrm{Si}$ process-etching around the Ge epitaxial layer on the Si substrate and selectively filling $\mathrm{Si}_{1-x} \mathrm{Ge}_{x}$ to properly introduce the biaxial tensile stress to realize the conversion of the Ge band gap type [25]. The structure of this method is shown in Figure 4 , where $d, T$, and $L$ correspond to the Ge mesa region width, $\mathrm{Si}_{1-x} \mathrm{Ge}_{x}$ stressor thickness, and the distance between adjacent mesas, respectively. This scheme is compatible with the Si process and relatively easy to implement, with the prospect of being a source material for MOEI.

Based on the theoretical model of the linear elastic material and COMSOL finite element analysis software, a finite element stress model is established by selecting the $\mathrm{Si}_{1-x} \mathrm{Ge}_{x}$ region in the Ge epitaxial layer on the Si substrate to implement this method. On the basis of the finite element physical model, the material physical and geometrical parameters of the $\mathrm{Si}_{1-x} \mathrm{Ge}_{x}$ selective growth region can be determined, which provides an important theoretical basis for the preparation process of the "direct band gap Ge implementation method compatible with the Si process."

\section{Finite Element Simulation Model}

The finite element method (FEM) is used to analyze the strain result from the lattice constant mismatch of semiconductor materials. Different virtual thermal expansion coefficients are usually set to simulate the distribution of stress or strain in materials caused by lattice mismatch [15]. In the simulation, it is assumed that the material properties are isotropic, in which case the strain effect caused by thermal expansion is very similar to that caused by the lattice constant mismatch $[26,27]$. Therefore, the strain caused by lattice mismatch between heterogeneous materials can be simulated by setting different thermal expansion coefficients for materials in the finite element simulation process.

Before the simulation analysis of the selective epitaxial modified Ge scheme, this section firstly uses the channel strain induced by selective epitaxy of the Si PMOS source and drain as the reference object to verify the correctness of the COMSOL finite element strain analysis software and the thermal expansion stress simulation method.

Based on the simulation principle of thermal expansion stress calculation mentioned above, the channel strain simulation of Si PMOS source-drain selective epitaxy is carried out. The expansion/contraction of the material due to temperature variation is the same as the strain due to lattice constants mismatch in practical devices, that is, $\Delta T \cdot C=f$, where $\Delta T$ is the temperature variation of the material, $C$ is the thermal expansion coefficient set manually, and $f$ is the lattice mismatch rate between heterogeneous materials. For the epitaxial SiGe layer on the Si substrate, the lattice mismatch rate $f$ can be obtained from the following equation:

$$
f=\frac{a_{\mathrm{Si}}-a_{\mathrm{SiGe}}}{a_{\mathrm{Si}}}=-0.0418 x,
$$

where the minus indicates that the Si material is subjected to compressive strain.

The heating method is adopted in the simulation process. Assuming a $1000^{\circ} \mathrm{C}$ rise in temperature, the virtual thermal expansion coefficient of $\mathrm{Si}_{0.5} \mathrm{Ge}_{0.5}$ is $2.09 \times 10^{-5} / \mathrm{K}$. The critical material parameters required for COMSOL simulation are listed in Table 1, and Young's modulus and 


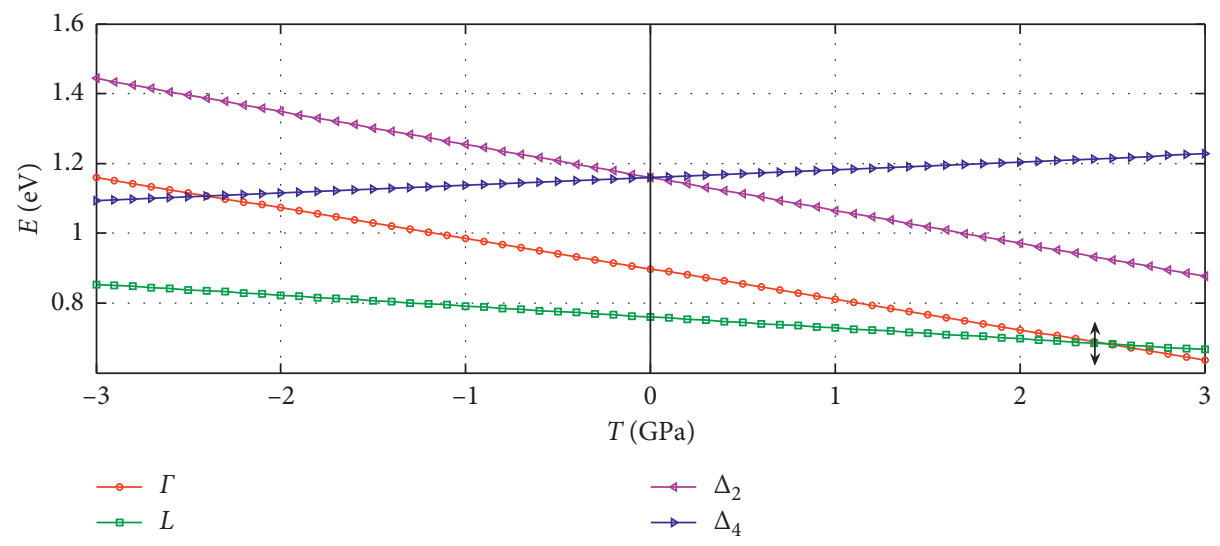

FIGURE 2: The variation of the energy levels of Ge conduction with the (001) biaxial tensile stress.

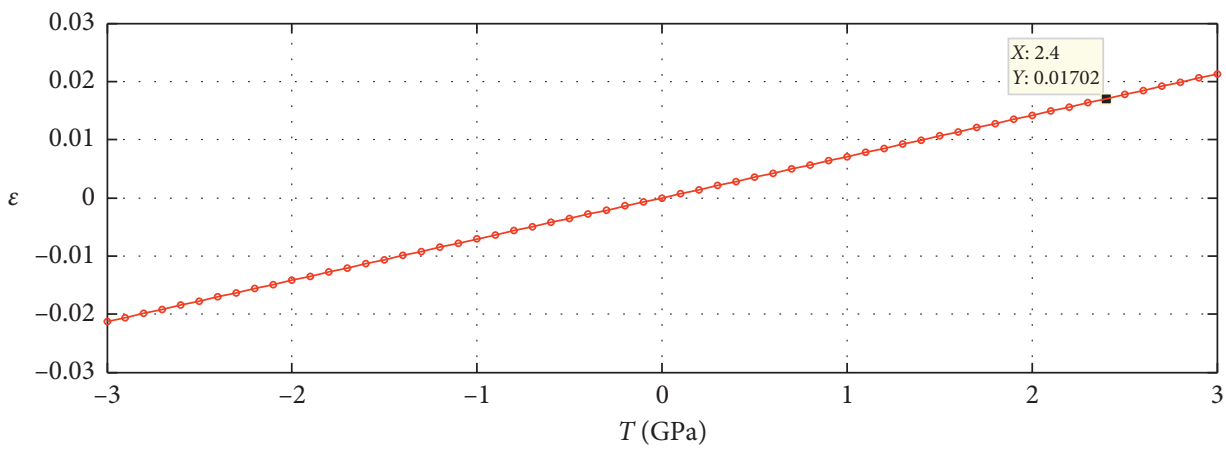

FIgURE 3: The variation of the strain generated with the (001) biaxial tensile stress.

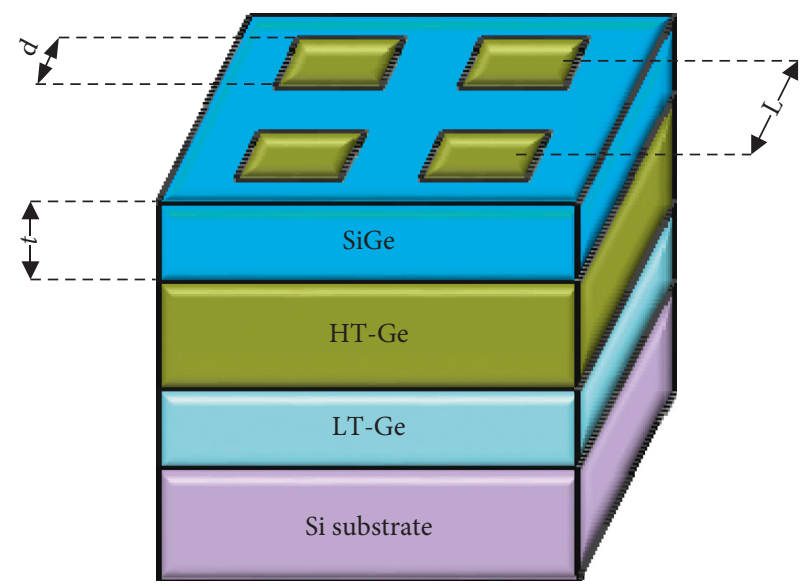

FIgURE 4: Structural diagram of the direct band gap Ge implementation method compatible with the Si process.

Poisson's ratio of the SiGe material are obtained by linear interpolation. The geometric parameters are as follows: the length of channel is $50 \mathrm{~nm}$, the depth of source and drain zone is $20 \mathrm{~nm}$, and the length of source and drain zone is $200 \mathrm{~nm}$.

After visualization of the results, the $\varepsilon_{x x}$ and $\varepsilon_{z z}$ strain distributions of the channel region are shown in Figure 5. Careful observation of the following Figure 5 shows that the distribution and magnitude of strain simulated by COMSOL software are in good agreement with the results obtained by
Yeo and Sun indicating that the method for studying strain distribution based on virtual thermal expansion is correct and feasible [28].

After verifying the correctness of COMSOL finite element analysis software and thermal expansion stress simulation method, next we modeled the selective epitaxyinduced direct band gap Ge scheme. The realization principle of this scheme is introduced first. Its preparation process is as shown in Figure 6.

Figure 7 shows the local three-dimensional (3D) structure and profile of the selective epitaxy-induced direct band gap Ge scheme, where $L$ is the width of the epitaxial layer, which is equal to the distance between adjacent mesas. Since the lattice constant of $\mathrm{Ge}$ is larger than that of $\mathrm{Si}_{1-x} \mathrm{Ge}_{x}$, the principle of lattice mismatch-induced stress is used to grow $\mathrm{Si}_{1-x} \mathrm{Ge}_{x}$ around the etched Ge mesa epitaxially, and the biaxial tensile stress will be introduced in the central region of $\mathrm{Ge}$ semiconductors.

According to the above structure diagram, a simulation model of selective epitaxy-induced direct band gap Ge is established based on the COMSOL simulation software. Because the force in the $X$ direction is identical to that in the $Y$ direction, and in order to obtain more precise simulation results, this section establishes a two-dimensional simulation model [21]. The modeling process is as follows: (1) analyze the problem and determine the simulation model; (2) create the geometric model; (3) set the material properties; (4) set the boundary conditions; (5) meshing; (6) solve and set the 
TABLE 1: COMSOL simulation parameters.

\begin{tabular}{lccc}
\hline Material category & Young's modulus $(\mathrm{GPa})$ & Virtual thermal expansion coefficient $\left(10^{-5} / \mathrm{K}\right)$ & Poisson's ratio \\
\hline $\mathrm{Si}$ & 162.91 & 0 & 0.28 \\
$\mathrm{Si}_{0.5} \mathrm{Ge}_{0.5}$ & 131.66 & 2.09 & 0.273 \\
\hline
\end{tabular}
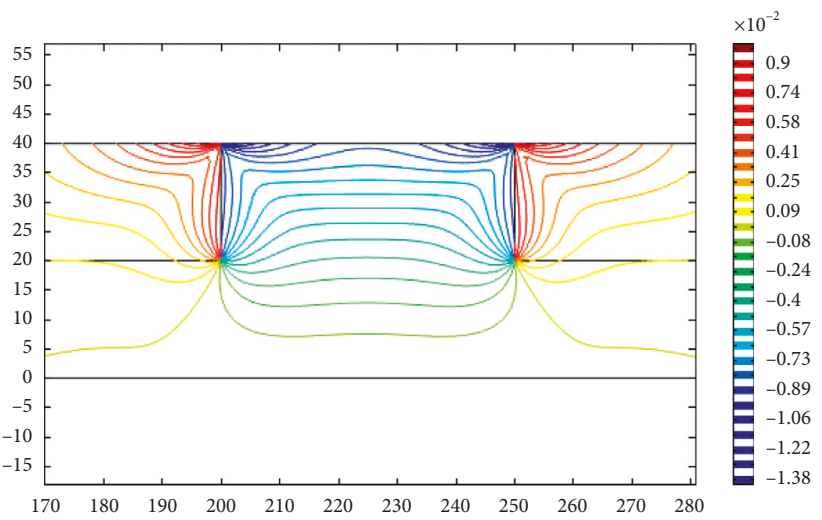

(a)

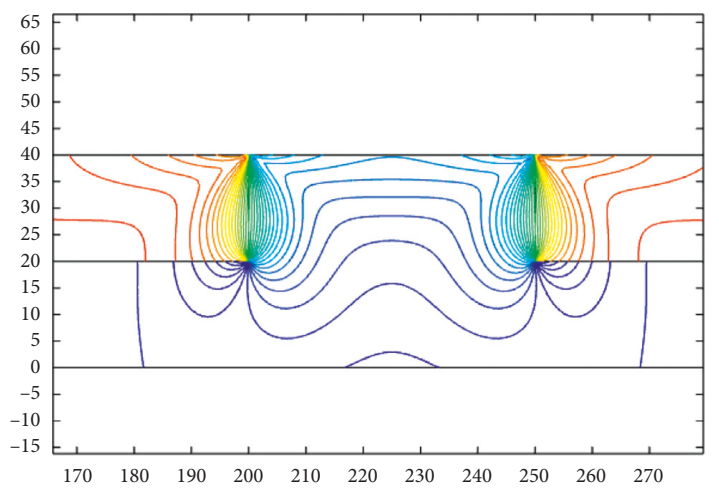

(c)

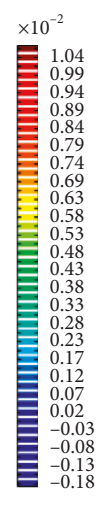

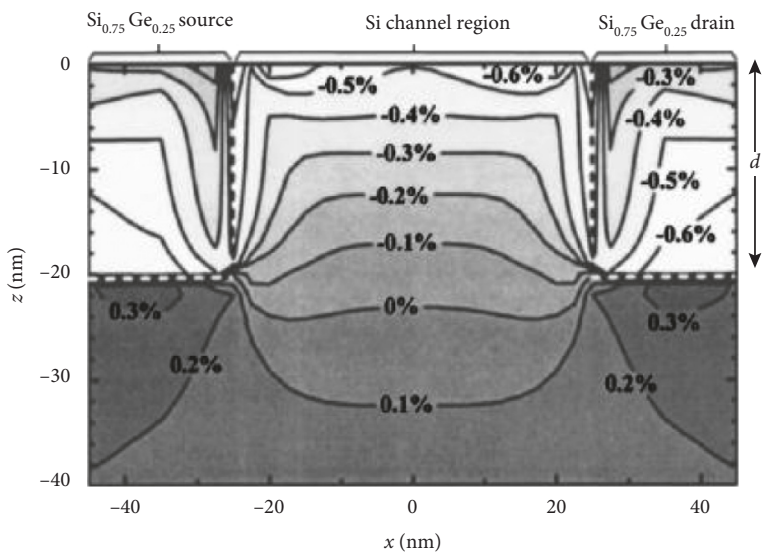

(b)

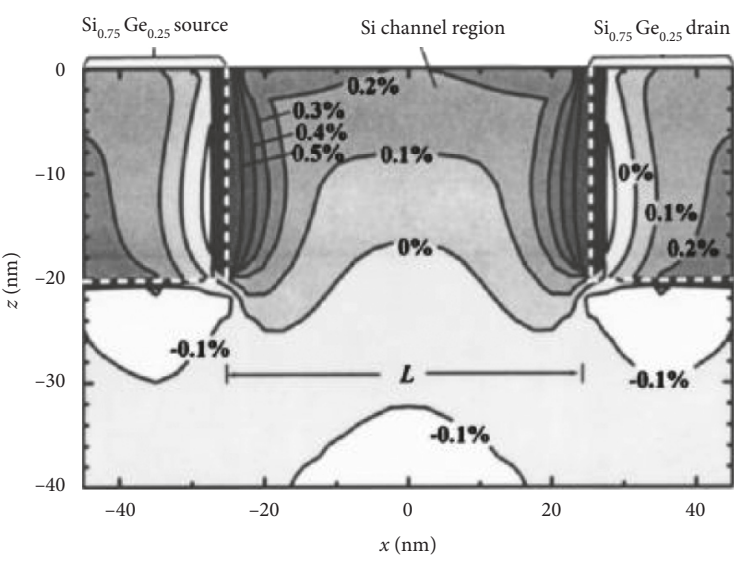

(d)

FIgURE 5: Channel strain simulation of Si PMOS source-drain selective epitaxy. (a) $\varepsilon_{x x}$ simulation result. (b) $\varepsilon_{x x}$ reference result. (c) $\varepsilon_{z z}$ simulation result. (d) $\varepsilon_{z z}$ reference result.

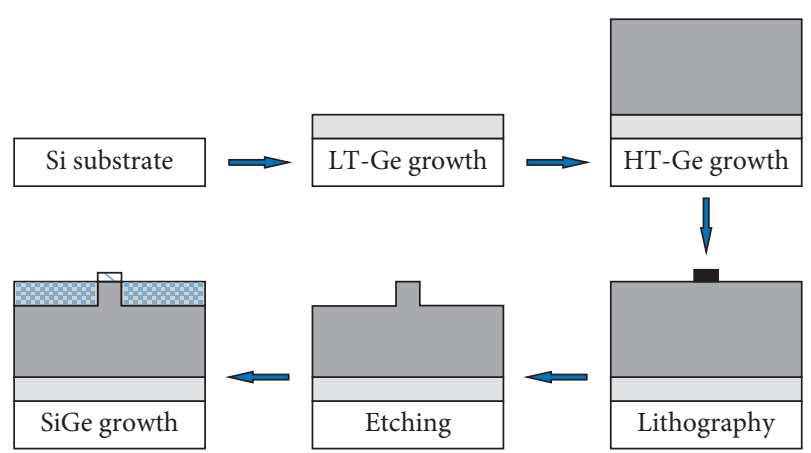

Figure 6: Preparation process of direct band gap Ge scheme compatible with the Si process.

solution parameters; (7) post processing and visualization of results.

Firstly, through the model wizard, the modeling options such as spatial dimension, physical field, and research type

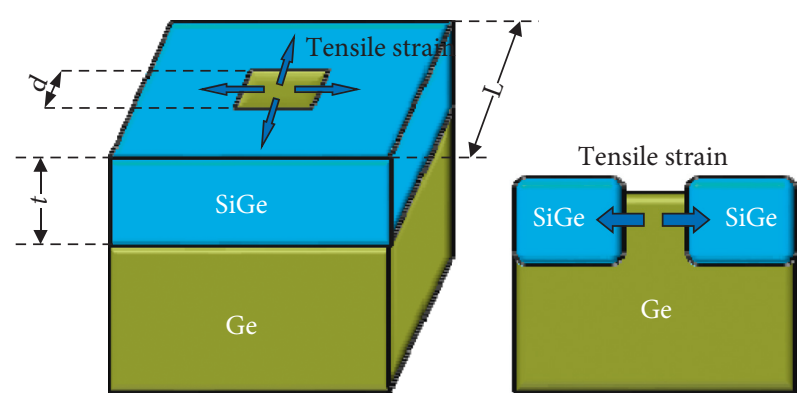

FIGURE 7: Local 3D structure and profile of selective epitaxy-induced direct band gap Ge scheme.

are built. The solid mechanics is selected in the module. After that, the corresponding geometric model is established and the material types and physical parameters in relevant regions are set. In the process, common Si and Ge Material Models in Semiconductor Material Library can be invoked, including basic material parameters, Shockley-Read-Hall 
composite model, band gap model, and other important models.

In this paper, the strain distribution is studied based on the thermal expansion method, so the thermal expansion model of the linear elastic material in the solid mechanics module is selected. The next most important step is to set the temperature load and boundary conditions: adding a preset temperature load to the thermal expansion model; subsequently, in order to simulate the real physical conditions, all the nodes on the bottom of the substrate are set as fixed constraints in the two-dimensional direction, and the other boundaries are free. Then, the selective epitaxy-induced direct band gap Ge model is meshed. Since the Ge mesa region is the key area of observation, the mesh for the Ge mesa region is densely divided, and the meshing effect is shown in Figure 8. Finally, the calculation type is selected to perform the operation; after the calculation result is completed, the data type is needed to set for image and drawing processing.

Both the composition of Ge in SiGe and parameters of the SiGe epitaxial layer will affect the strain distribution and magnitude in the mesa; therefore, the influence of the variation of the simulation parameters on the strain in $\mathrm{Ge}$ mesa is studied by the strain situation in the $X$ direction.

\section{Results and Discussion}

3.1. The Effect of Width of the Epitaxial Layer on the Strain. Referring to the height of the source and drain regions in the Si PMOS channel strain, the Ge step width is set to $20 \mathrm{~nm}$, the Ge composition is taken as 0.5 , and the strain situation is analyzed by changing the width of the epitaxial layer. The width of the epitaxial layer is changed from $40 \mathrm{~nm}$ to $200 \mathrm{~nm}$, and the strain value at $5 \mathrm{~nm}$ in the Ge mesa is as shown by the red curve in Figure 9. From the graph, it can be seen that the strain increases linearly with the increase in the width of the SiGe epitaxial layer when the width is less than $100 \mathrm{~nm}$. However, when the width of the epitaxial layer is 140 $160 \mathrm{~nm}$, the strain varies slightly with the increase in the width and tends to be stable gradually; that is, the tensile strain no longer changes as the width of the epitaxial layer increases.

It is possible to obtain the epitaxial layer size for stable strain under different step widths by setting the Ge step width to $25 \mathrm{~nm}, 30 \mathrm{~nm}, 35 \mathrm{~nm}$, and $40 \mathrm{~nm}$, respectively. And through a series of simulation experiments, the relationship between the width of the epitaxial layer for a stable strain value and the Ge step width is determined, as shown in Figure 10. It can be found that there is a linear relationship between them. The fitted formula is $y=5.1 x+52$ ( $x$ is the step width, $y$ is the width of the epitaxial layer, and the unit is nanometer).

3.2. The Effect of Ge Composition on the Strain. The theoretical analysis shows that as the Ge composition decreases, the stress in the Ge step increases. The reason is that the lattice constant of the epitaxial layer decreases with the decrease of $\mathrm{Ge}$ composition and the lattice constant

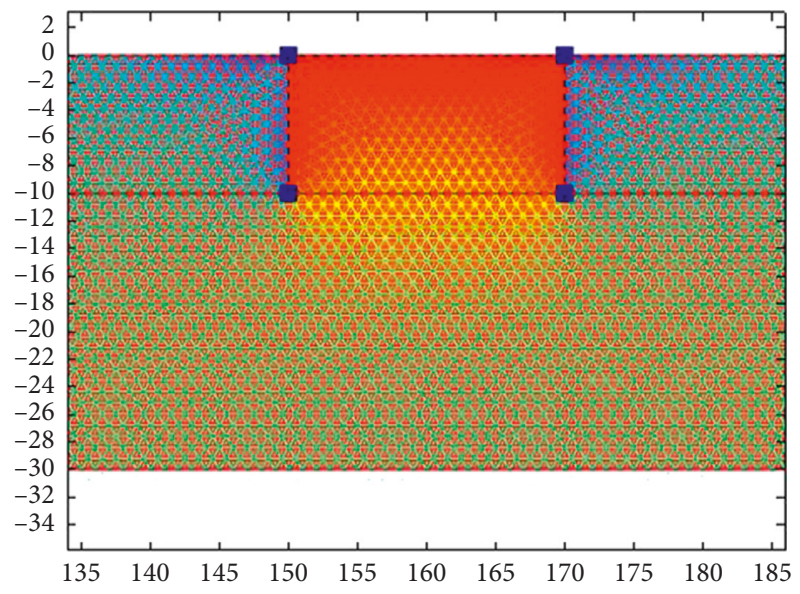

FIgURE 8: Meshing diagram of the Ge mesa region.

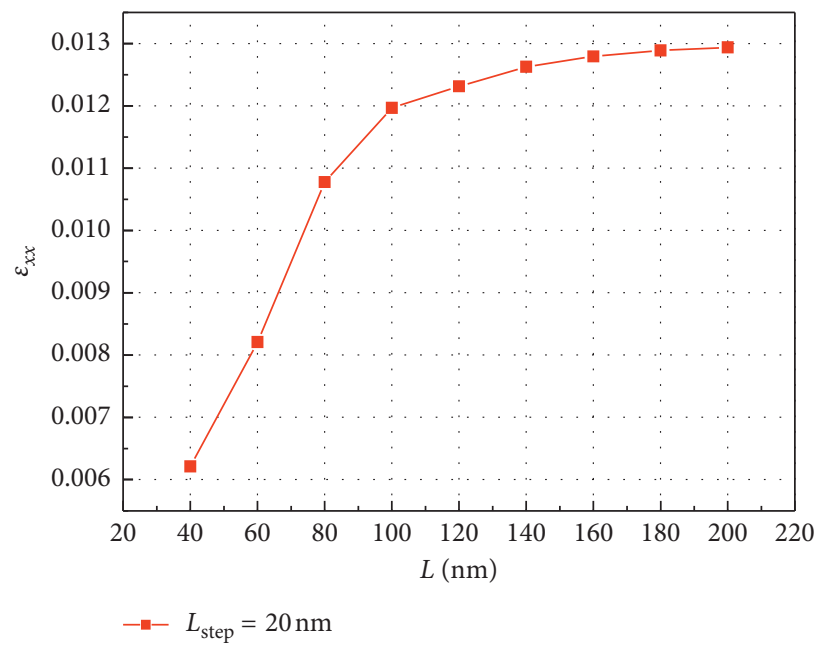

FIgURE 9: Variation of strain with the width of the epitaxial layer when the width of the Ge step is $20 \mathrm{~nm}$.

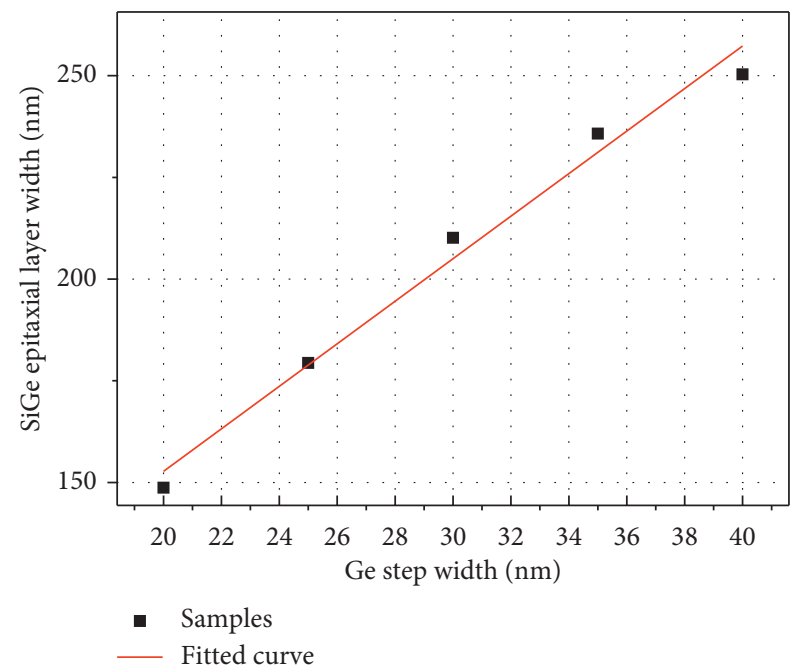

FIgURE 10: Variation of the SiGe epitaxial layer width for stable strain with the Ge step width. 
TABLe 2: Simulation parameters of the SiGe material under different Ge components.

\begin{tabular}{lccc}
\hline Ge components & Young's modulus $(\mathrm{GPa})$ & Virtual thermal expansion coefficient $\left(10^{-5} / \mathrm{K}\right)$ & Poisson's ratio \\
\hline 0.2 & 156.6 & -3.21 & 0.2786 \\
0.3 & 153.5 & -2.81 & 0.2779 \\
0.4 & 150.4 & -2.41 & 0.2772 \\
0.5 & 147.3 & -2.0 & 0.2765 \\
\hline
\end{tabular}

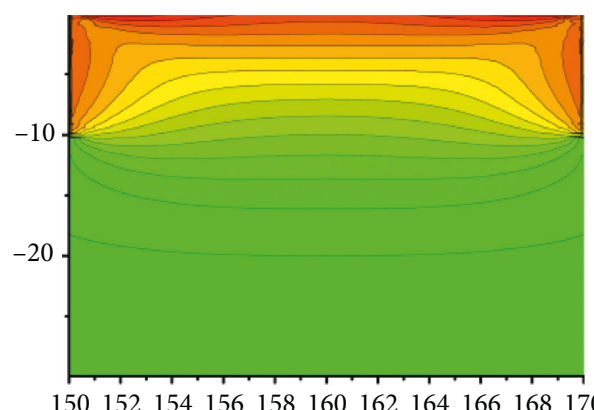

(a)

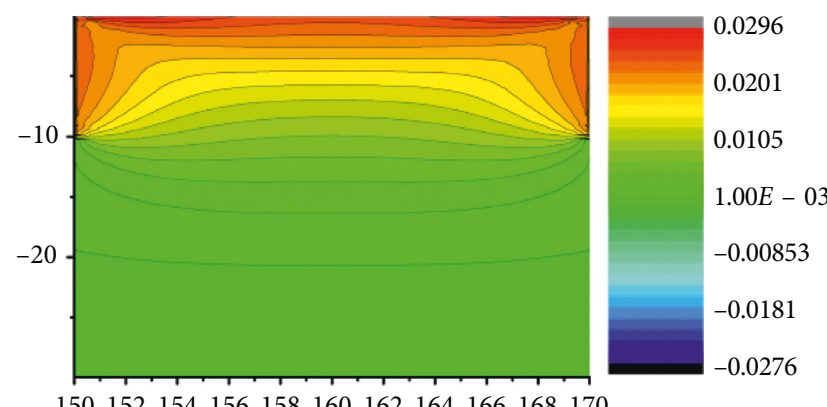

150152154156158160162164166168170

(c)

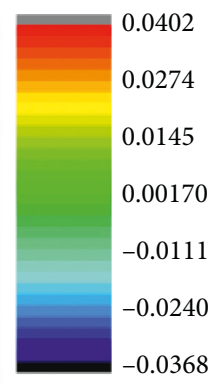

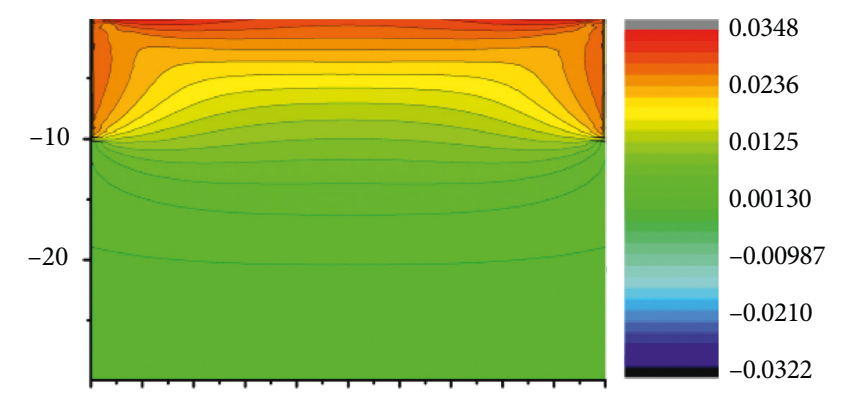

150152154156158160162164166168170

(b)

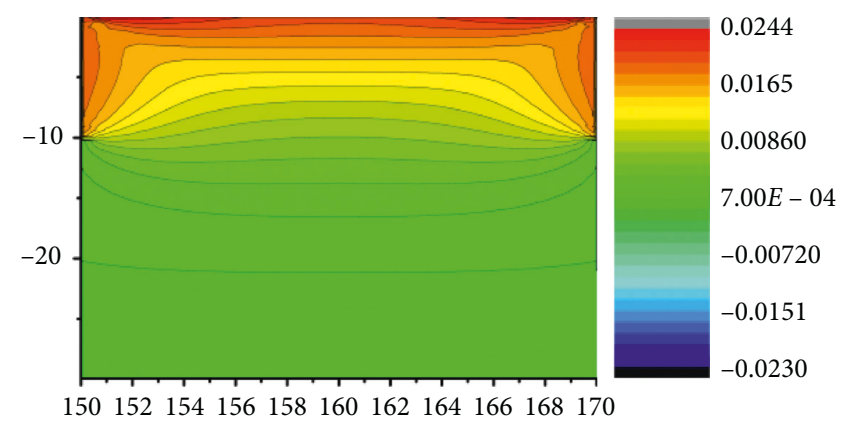

(d)

Figure 11: Distribution of $\varepsilon_{x x}$ in the mesa region when the Ge component is $0.2,0.3,0.4$, and 0.5 .

difference between the Ge and SiGe epitaxial layers becomes larger, so does the introduced tension strain. Keeping the parameters of Ge step width and epitaxial layer width unchanged, the Ge component in the SiGe epitaxial layer increased from 0.2 to 0.5 and the relationship between the $\mathrm{Ge}$ component and the $X$-direction strain can be obtained [29]. Table 2 shows the material parameters of the $\mathrm{Si}_{1-x} \mathrm{Ge}_{x}$ epitaxial layer under different Ge components. Young's modulus and Poisson's ratio are calculated by linear interpolation, while the virtual thermal expansion coefficient is calculated from the lattice mismatch rate.

The Ge step width is fixed at $20 \mathrm{~nm}$, and the Ge component is selected to be $0.2,0.3,0.4$, and 0.5 . From the analysis in the previous section, it can be seen that if the width of the Ge step is $20 \mathrm{~nm}$, the maximum strain value can be achieved when the width of the SiGe epitaxial layer is set to about $150 \mathrm{~nm}$. Therefore, the width of the SiGe epitaxial layer is set to $150 \mathrm{~nm}$ in the simulation. The obtained simulation results are shown in Figure 11. The strain distribution in the four images is basically the same, just that there are differences in the magnitude of the strain.

Figure 12 shows the horizontal distribution of $\varepsilon_{x x}$ at a depth of $5 \mathrm{~nm}$ from the mesa's surface. It can be seen that when the Ge component is 0.3 , the strain of the Ge material at $5 \mathrm{~nm}$ depth has all reached $1.7 \%$ or more, which can realize the transition from the indirect band gap to direct band gap. But when the Ge component continues to increase, such as 0.4 , only parts of the strain near the boundary in the mesa exceed $1.7 \%$.

Figure 13 shows the depths where the strains of the Ge central region equal to $1.7 \%$ under different components. For example, when $x=0.3$, the depth at which all the central regions meet the requirement of band gap transition is $5.775 \mathrm{~nm}$. From the figure and the analysis above, it can be seen that the Ge component has a great influence on the strain in the mesa region, and reducing the Ge component can greatly increase the strain. However, if the Ge component is too small, the lattice mismatch between the SiGe epitaxial layer and Ge material will be too large, which is disadvantageous for preparing a high-quality $\mathrm{SiGe}$ epitaxial layer. Therefore, the $\mathrm{Ge}$ component is selected to be $0.3-0.5$. In this range, not only enough strain can be obtained for the Ge material but also the quality of the SiGe film is relatively good, which is conducive to the preparation of the modified Ge devices.

3.3. The Effect of Ge Step Thickness on the Strain. The Ge component is 0.5 , the width of the epitaxial layer is $L=150 \mathrm{~nm}$, and the length and width of the step is $20 \mathrm{~nm}$. 


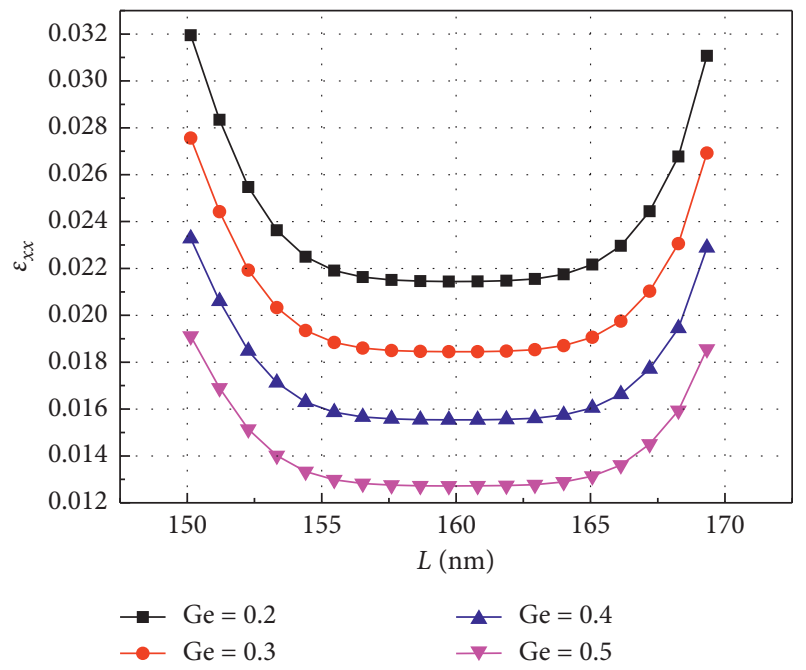

FIgURE 12: Magnitude regularity of $\varepsilon_{x x}$ at $5 \mathrm{~nm}$ depth when the Ge component is $0.2,0.3,0.4$, and 0.5 .

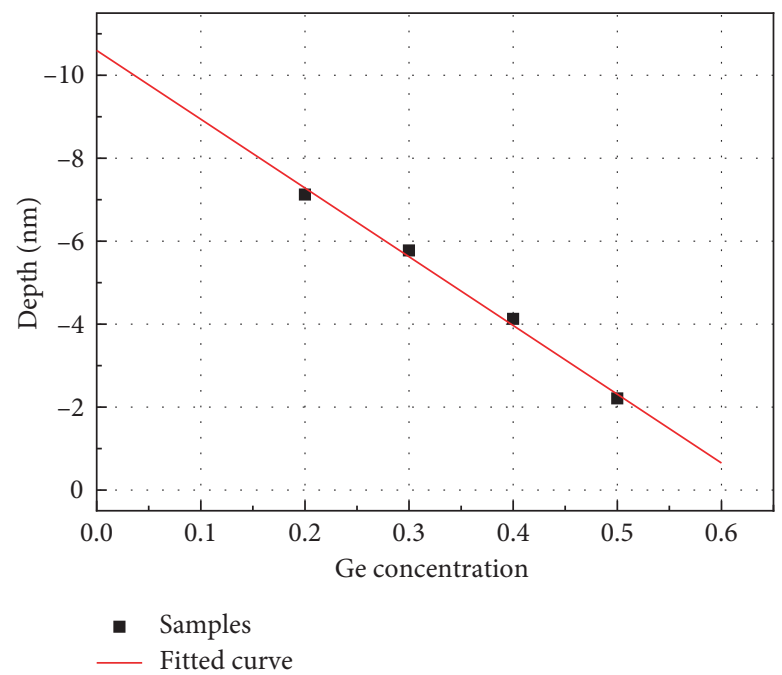

Figure 13: Depth that band gap transition can be achieved under different Ge components.

The influence of SiGe layer thickness on strain tensor of the Ge material is simulated by taking the thickness of the Ge step as $10,20,30$, and $40 \mathrm{~nm}$. The simulation results are shown in Figure 14.

The simulation results indicate that changing the thickness of the Ge step has little effect on the distribution and magnitude of the strain generated in the Ge step, which can be neglected.

3.4. Verification of Conclusions. Through the above analysis, the influence of the width of the epitaxial layer and the Ge component in the $\mathrm{Si}_{1-x} \mathrm{Ge}_{x}$ epitaxial layer on the strain magnitude and distribution in the Ge step can be obtained. Then, the final optimization scheme of selective epitaxy-induced direct band gap Ge is proposed: Ge step size is $20-40 \mathrm{~nm}$, SiGe epitaxial layer width is $150 \sim 250 \mathrm{~nm}$, and Ge component in the epitaxial layer is $0.3-0.5$, which can realize the direct band gap Ge material in the surface of the mesa region. In this paper, the

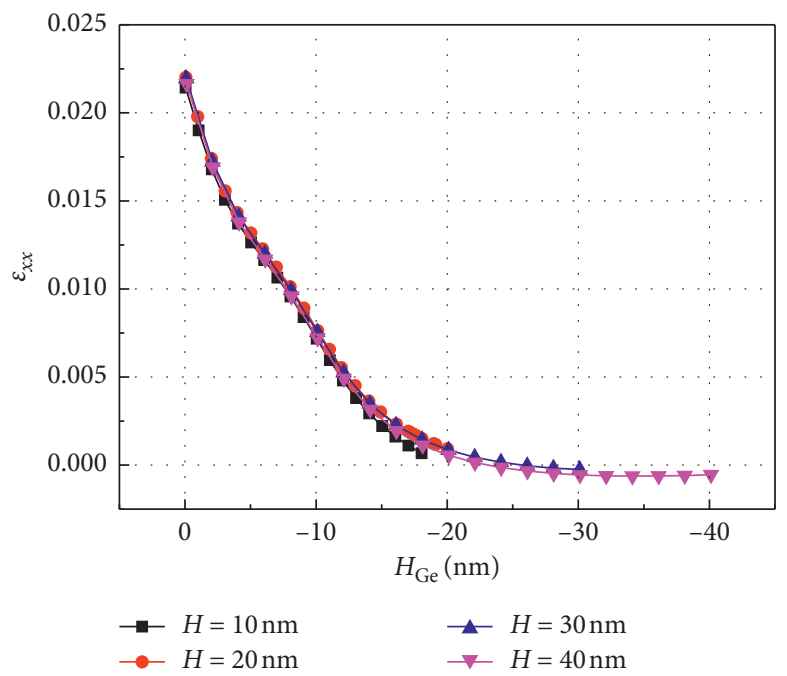

Figure 14: Vertical strain distribution at different step thicknesses. 


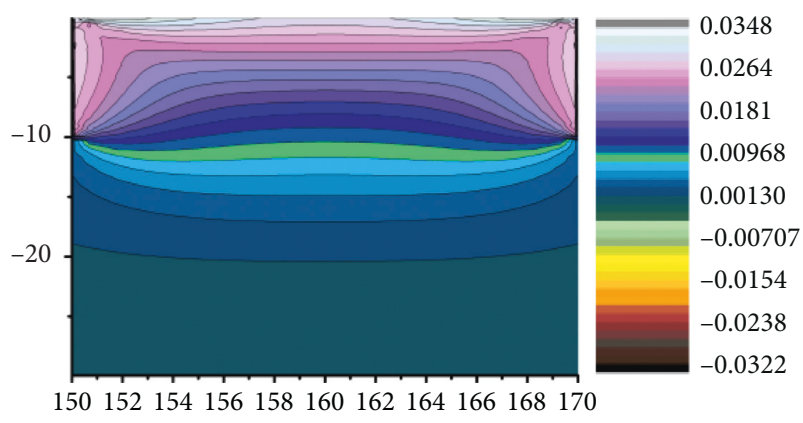

(a)

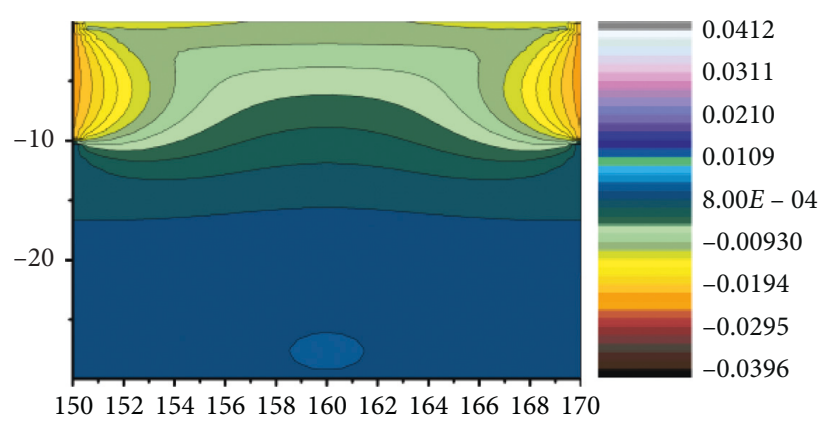

(b)

FIgURE 15: Contour map of $\varepsilon_{x x}$ and $\varepsilon_{z z}$ in the Ge mesa area.

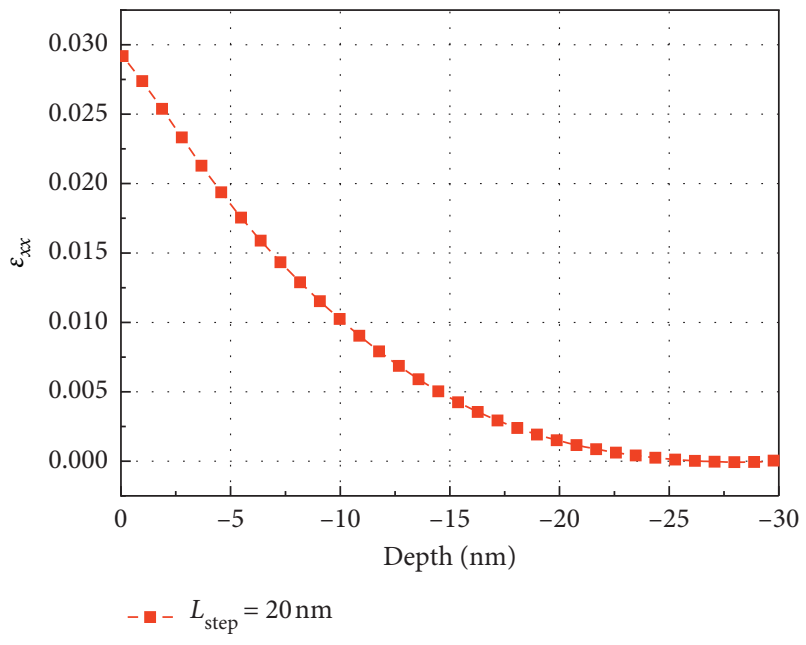

FIgURE 16: Vertical change diagram of $\varepsilon_{x x}$ in the Ge mesa area.

width of the Ge step is $20 \mathrm{~nm}$, the thickness is $10 \mathrm{~nm}$, the width of the SiGe epitaxial layer is $210 \mathrm{~nm}$, and the Ge component is 0.3 . The strain distribution in the Ge step is simulated by the thermal expansion simulation model. Figure 15 is a contour map of $\varepsilon_{x x}$ and $\varepsilon_{z z}$ in the Ge mesa area.

From Figure 16, the distribution trend of stress in the step can be seen. The stress near the SiGe epitaxial layer on both sides of the mesa is larger, while the stress near the center of the mesa is smaller, and the strain in the horizontal direction is more uniform. In the vertical direction, the strain decreases rapidly with the increase in the distance from the mesa surface. Figure 16 shows this law more intuitively, at $X=0 \mathrm{~nm}, \varepsilon_{x x}$ is close to $3 \%$, and when $X=-10 \mathrm{~nm}$, the strain is less than $1 \%$. At the center, the depth that its tensile strain exceeds $1.7 \%$ is close to $6 \mathrm{~nm}$.

Because the internal strains in the Ge step are different and the strains generated on the surface of the step and its vicinity are higher than those in other regions, these simulation results fully prove that the direct band gap transition of the Ge material can be achieved in some regions of the step by optimizing the model parameters.

\section{Conclusion}

Focusing on the "the implementation method of direct band gap Ge compatible with the Si process," this paper uses the finite element analysis technique to establish a finite element stress model for selectively a growing $\mathrm{Si}_{1-x} \mathrm{Ge}_{x}$ region-induced Ge band gap transition and analyze the material physical parameters, geometric structure parameters, and stress distribution required for Ge to achieve direct band gap transition.

The results show that the strains near the SiGe epitaxial layer and distributed on both sides of the Ge step are larger, while the strains generated near the center of the step are smaller, the strains in the horizontal direction of the step center are relatively uniform, and the strains in the vertical direction decrease rapidly with the increase in the distance from the mesa surface. The magnitude of the strain in the Ge material can be adjusted according to the Ge composition and the width of the epitaxial layer. The geometric dimension of the Ge center region is fixed, and the strain in the Ge material increases linearly with the increase in SiGe epitaxial layer width around and tends to be stable. The smaller the Ge component in the $\mathrm{Si}_{1-x} \mathrm{Ge}_{x}$ epitaxial layer, the larger the area of the direct band gap Ge semiconductor. When the $\mathrm{Si}_{1-x} \mathrm{Ge}_{x}$ epitaxial layer is $150-250 \mathrm{~nm}$ wide and the Ge composition is $0.3 \sim 0.5$, Ge mesa with $20-40 \mathrm{~nm}$ in width can be transformed into the direct band gap semiconductor in the depth of $0-6 \mathrm{~nm}$.

The physical and geometrical parameters of the $\mathrm{Si}_{1-x} \mathrm{Ge}_{x}$ selective growth region are obtained based on the theoretical results, which can provide an important theoretical basis for the realization of subsequent related processes.

\section{Data Availability}

All data of this paper used to support the findings of this study are available from the corresponding author upon request.

\section{Conflicts of Interest}

The authors declare that there are no conflicts of interest regarding the publication of this paper.

\section{Acknowledgments}

This work was supported by the 111 Project (Grant no. B12026). 


\section{References}

[1] Y. Huo, H. Lin, R. Chen et al., "Strong enhancement of direct transition photoluminescence with highly tensile-strained Ge grown by molecular beam epitaxy," Applied Physics Letters, vol. 98, no. 1, article 011111, 2011.

[2] G. E. Moore, "Cramming more components onto integrated circuits," Proceedings of the IEEE, vol. 86 , no. 1, pp. 82-85, 1998.

[3] C. Sun, M. T. Wade, Y. Lee et al., "Single-chip microprocessor that communicates directly using light," Nature, vol. 528, no. 7583, pp. 534-538, 2015.

[4] A. H. Atabaki, S. Moazeni, F. Pavanello et al., "Integrating photonics with silicon nanoelectronics for the next generation of systems on a chip," Nature, vol. 556, no. 7701, pp. 349-354, 2018.

[5] R. Won and M. Paniccia, "Integrating silicon photonics," Nature Photonics, vol. 4, no. 8, pp. 498-499, 2010.

[6] G. Sun, H. H. Cheng, J. Menéndez, J. B. Khurgin, and R. A. Soref, "Strain-free $\mathrm{Ge} / \mathrm{GeSiSn}$ quantum cascade lasers based on $L$-valley intersubband transitions," Applied Physics Letters, vol. 90, no. 25, Article ID 251105, 2007.

[7] J. F. Liu, X. C. Sun, D. Pan et al., "Tensile-strained, n-type Ge as a gain medium for monolithic laser integration on $\mathrm{Si}$," Optics Express, vol. 15, no. 18, p. 11272, 2007.

[8] R. A. Minamisawa, M. J Süess, R Spolenak et al., "Top-down fabricated silicon nanowires under tensile elastic strain up to 4.5\%," Nature Communications, vol. 3, no. 1, p. 1096, 2012.

[9] M. J. Süess, R. Geiger, R. A. Minamisawa et al., "Analysis of enhanced light emission from highly strained germanium microbridges," Nature Photonics, vol. 7, no. 6, pp. 466-472, 2013.

[10] D. Sukhdeo, D. Nam, J.-H. Kang, M. L. Brongersma, and K. C. Saraswa, "Direct bandgap germanium-on-silicon inferred from 5.7\% uniaxial tensile strain," Photonics Research, vol. 2, no. 3, pp. A8-A13, 2014.

[11] J. Petykiewicz, D. Nam, D. S. Sukhdeo et al., "Direct bandgap light emission from strained germanium nanowires coupled with high-Q nanophotonic cavities," Nano Letters, vol. 16, no. 4, pp. 2168-2173, 2016.

[12] D. Nam, D. Sukhdeo, A. Roy et al., "Strained germanium thin film membrane on silicon substrate for optoelectronics," Optics Express, vol. 19, no. 27, pp. 25866-25872, 2011.

[13] D. S. Sukhdeo, J. Petykiewicz, S. Gupta et al., "Ge microdisk with lithographically-tunable strain using CMOS-compatible process," Optics Express, vol. 23, no. 26, p. 33249, 2015.

[14] A. Elbaz, M. E. Kurdi, A. Aassime et al., "Germanium microlasers on metallic pedestals," APL Photonics, vol. 3, no. 10, Article ID 106102, 2018.

[15] S. Wirths, R. Geiger, N. von den Driesch et al., "Lasing in direct-bandgap GeSn alloy grown on Si," Nature Photonics, vol. 9, no. 2, pp. 88-92, 2015.

[16] S. Bao, D. Kim, C. Onwukaeme et al., "Low-threshold optically pumped lasing in highly strained germanium nanowires," Nature Communications, vol. 8, no. 1, pp. 1-7, 2017.

[17] J. Mathews, R. Roucka, J. Xie, S.-Q. Yu, J. Menéndez, and J. Kouvetakis, "Extended performance $\mathrm{GeSn} / \mathrm{Si}$ (100) $p-i-n$ photodetectors for full spectral range telecommunication applications," Applied Physics Letters, vol. 95, no. 13, Article ID 133506, 2009.

[18] G. Sun, R. A. Soref, and H. H. Cheng, "Design of a Si-based lattice-matched room-temperature $\mathrm{GeSn} / \mathrm{GeSiSn}$ multiquantum-well mid-infrared laser diode," Optics Express, vol. 18, no. 19, pp. 19957-19965, 2010.
[19] M. V. Fischetti and S. E. Laux, "Band structure, deformation potentials, and carrier mobility in strained $\mathrm{Si}, \mathrm{Ge}$, and $\mathrm{SiGe}$ alloys," Journal of Applied Physics, vol. 80, no. 4, pp. 22342252, 1996.

[20] T. Tsukamoto, N. Hirose, A. Kasamatsu, T. Mimura, T. Matsui, and Y. Suda, "Formation of GeSn layers on Si (001) substrates at high growth temperature and high deposition rate by sputter epitaxy method," Journal of Materials Science, vol. 50, no. 12, pp. 4366-4370, 2015.

[21] S. Gupta, B. Magyari-Köpe, Y. Nishi, and K. C. Saraswat, "Achieving direct band gap in germanium through integration of Sn alloying and external strain," Journal of Applied Physics, vol. 113, no. 7, Article ID 073707, 2013.

[22] D. W. Jenkins and J. D. Dow, "Electronic properties of metastable $\mathrm{Ge}_{x} \mathrm{Sn}_{1-x}$ alloys," Physical Review B, vol. 36, no. 15, pp. 7994-8000, 1987.

[23] P. Moontragoon, Z. Ikonić, and P. Harrison, "Band structure calculations of Si-Ge-Sn alloys: achieving direct band gap materials," Semiconductor Science and Technology, vol. 22, no. 7, pp. 742-748, 2007.

[24] Y. Chibane, B. Bouhafs, and M. Ferhat, "Unusual structural and electronic properties of $\mathrm{Sn}_{x} \mathrm{Ge}_{1-x}$ alloys," Physica Status Solidi (B), vol. 240, no. 1, pp. 116-119, 2003.

[25] Y. Hoshina, K. Iwasaki, A. Yamada, and M. Konagai, "Firstprinciples analysis of indirect-to-direct band gap transition of Ge under tensile strain," Japanese Journal of Applied Physics, vol. 10, no. 4, 2008.

[26] S. C. Jain, A. H. Harker, A. Atkinson, and K. Pinardi, "Edgeinduced stress and strain in stripe films and substrates: a twodimensional finite element calculation," Journal of Applied Physics, vol. 78, no. 3, pp. 1630-1637, 1995.

[27] T. Benabbas, Y. Androussi, and A. Lefebvre, "A finite-element study of strain fields in vertically aligned InAs islands in GaAs," Journal of Applied Physics, vol. 86, no. 4, pp. 19451950, 1999.

[28] Y.-C. Yeo and J. Sun, "Finite-element study of strain distribution in transistor with silicon-germanium source and drain regions," Applied Physics Letters, vol. 86, no. 2, Article ID 023103, 2005.

[29] G. R. Liu and S. S. Q. Jerry, "A finite element study of the stress and strain fields of InAs quantum dots embedded in GaAs," Semiconductor Science and Technology, vol. 17, no. 6, pp. 630-643, 2002. 

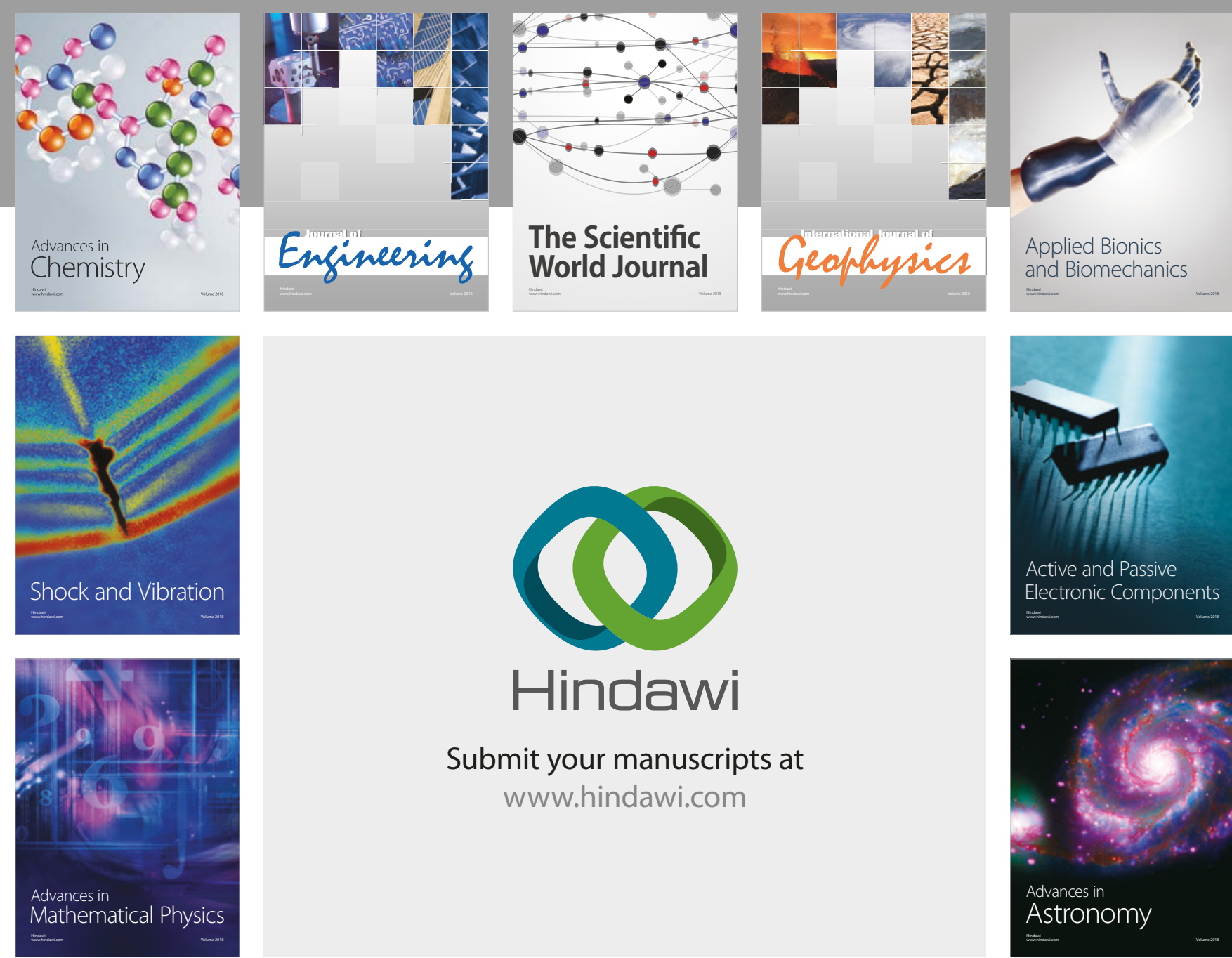

Submit your manuscripts at

www.hindawi.com

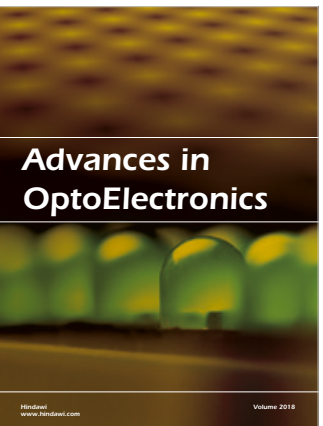

\section{Rotcting Machinery}
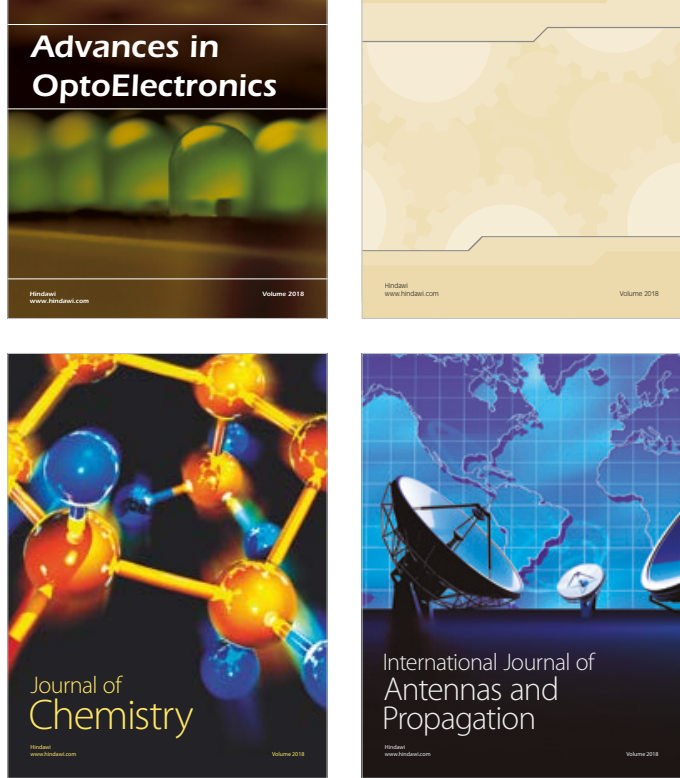

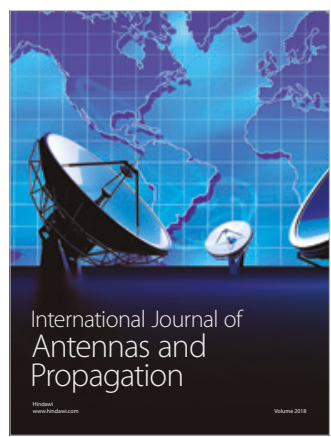

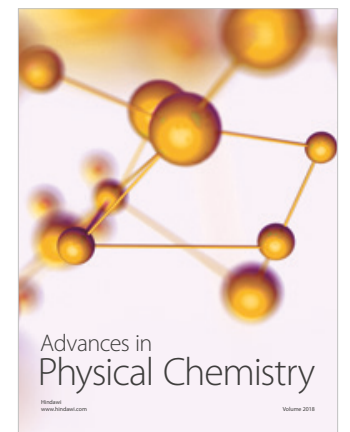

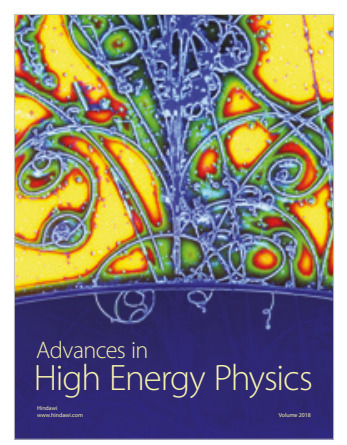

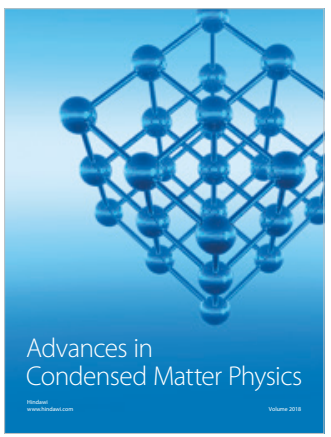

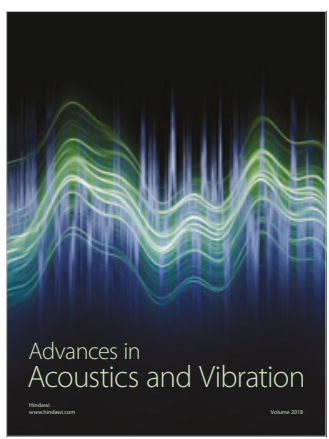

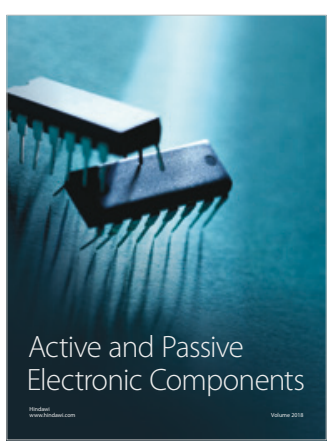
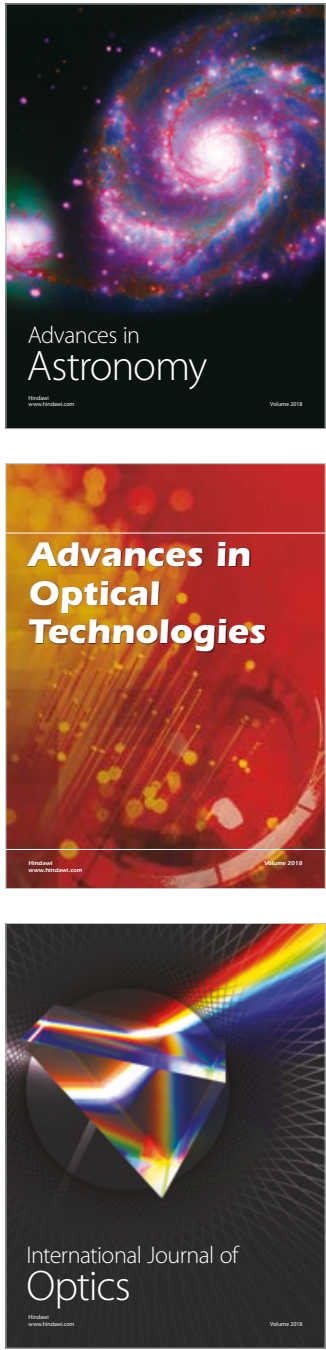